\title{
Evaluación de Competencias Informacionales con el Instrumento IL-HUMASS: Escalamiento Multidimensional
}

\author{
Information Literacy Evaluation with IL-HUMASS Scale: Multidimensional Scaling
}

\author{
Juan Pablo Hernández $\operatorname{Ramos}^{1}$, Fernando Martínez $\mathrm{Abad}^{2}$, Susana Olmos Migueláñez ${ }^{3}$, Maria José \\ Rodríguez Conde ${ }^{4}$
}

\section{Resumen}

En este trabajo se muestra el comportamiento multivariante de la escala IL-HUMASS, compuesta de 26 ítems de escala tipo Likert y 4 dimensiones teóricas, para la evaluación del nivel autopercibido en competencias informacionales. Se aplica la escala a una muestra de 402 sujetos, futuros profesores de educación secundaria en España. La técnica de escalamiento multidimensional mediante ALSCAL, muestra dos dimensiones de máxima explicación que explican el 95.9\% de la varianza de la matriz. Las dimensiones tienen que ver con la familiaridad con el desempeño propuesto en el ítem en primer lugar, y con el componente instrumental o cognitivo del ítem, en segundo lugar. Se concluye relacionando estas dos dimensiones con las dimensiones teóricas de la escala inicial. En las conclusiones se señala la importancia de tener en cuenta las dos dimensiones obtenidas, además de las dimensiones teóricas señaladas, a la hora de diseñar escalas relacionadas con la evaluación de competencias informacionales.

Palabras clave: alfabetización informacional, escalamiento multidimensional, análisis psicométrico, educación secundaria

\begin{abstract}
The multivariate characteristics of the IL-HUMASS scale are studied. This scale is formed by 26 Likert scale items and 4 dimensions and evaluate the information literacy self-perceived level of students. A sample of 402 subjects, all of them Spanish future teachers from secondary education, is obtained. It is implemented a multidimensional scaling technique by ALSCAL based on the distance matrix from the data. The results detected two empirical dimensions, which accounted for $95.9 \%$ of the variance. The first dimension is related to the level of familiarity with the content of the item and the second dimension is related to cognitive or instrumental aspect of the item. In the conclusions, two dimensions obtained are connected with the original scale, in relation to the four theoretical dimensions. On the other hand, the conclusions highlight the importance of considering the two dimensions obtained, in addition to the classical dimensions, in the design of assessment scales of the construct information literacy.
\end{abstract}

Keywords: information literacy, multidimensional scaling, psychometric analysis, secondary education

\footnotetext{
${ }^{1}$ Doctor en Ciencias de la Educación y Profesor Asociado al Dpto. de Didáctica, Organización y Métodos de Investigación. Universidad de Salamanca. Paseo de Canalejas, 169- 37008 Salamanca. Telf.: 923294630-3422. juanpablo@usal.es

${ }^{2}$ Doctor en Ciencias de la Educación y Profesor Ayudante del Área de Métodos de Investigación y Diagnóstico en Educación. Facultad de Educación. Universidad de Salamanca. Paseo de Canalejas, 169- 37008 Salamanca. Telf.: 923294630-3422. fma@usal.es

${ }^{3}$ Doctora en Ciencias de la educación y Profesora Ayudante Doctor del Área de Métodos de Investigación y Diagnóstico en Educación. Facultad de Educación. Universidad de Salamanca. Paseo de Canalejas, 169- 37008 Salamanca. Telf.: 9232946303406.solmos@usal.es

${ }^{4}$ Doctora en Ciencias de la Educación y Profesora Titular de Universidad de Métodos de Investigación Educativa. Facultad de Educación. Universidad de Salamanca. Paseo Canalejas, 169, 37008 Salamanca. Telf: 923294630-3442
} 


\section{Introducción}

El desarrollo técnico impulsado en la sociedad actual ha modificado completamente nuestra relación con el entorno y con el resto de sujetos (Castells, 1999). Las tecnologías asociadas a este contexto postindustrial potencian buena parte de las capacidades del hombre (Prensky, 2009); no sólo las que tienen que ver con aquellas capacidades sensoriales, sino también con otras más complejas, del ámbito cognitivo (McLuhan, 1987).

Así, debido a estos cambios, sociólogos y estudiosos de la realidad social comienzan a demandar una nueva denominación de esta "sociedad", más acorde a las nuevas circunstancias que la rodean (Cassity \& Ang, 2006; Cebrián Herreros, 2009; Valimaa \& Hoffman, 2008). La denominación que se ha generalizado en la literatura científica y que ha suscitado más adeptos es la de "sociedad de la información" (Area Moreira, 2001; García Carrasco, 2009).

En este contexto totalmente novedoso el mundo de la educación, cuyas bases fundadoras están fundamentadas en una sociedad industrial, trata de dar una respuesta adaptativa (Martorell, Gónzalez, Aloy, \& Ferris, 1995). Es por eso que surgen instituciones, tanto a nivel nacional como internacional, que hacen virar el paradigma educativo hacia la formación basada en competencias (Delors, 1997; Diario oficial de la Unión Europea, 2006; OCDE, 2004; UNESCO, 2005).

Se pueden definir estas competencias clave o básicas como: «Una combinación de conocimientos, capacidades y actitudes adecuadas al contexto. Las competencias clave son aquellas que todas las personas precisan para su realización y desarrollo personales, así como para la ciudadanía activa, la inclusión social y el empleo» (Diario oficial de la Unión Europea, 2006, p. 13)

En este entorno de formación basada en competencias básicas se puede identificar una competencia clave o básica, la competencia informacional, que se identifica con la denominada competencia para el tratamiento de la información y competencia digital de los documentos reguladores españoles y con la competencia digital de los documentos reguladores europeos.

En base al estudio de la Comisión sectorial de Tecnologías de la Información y las Comunicaciones de la Conferencia de Rectores de Universidades Españolas (CRUE-TIC) junto con la Red de Bibliotecas Universitarias (REBIUN), se puede proponer una definición de competencia informacional como la que sigue.

«Las competencias informacionales son el conjunto de conocimientos, habilidades, disposiciones y conductas que capacitan a los individuos para reconocer cuándo necesitan información, dónde localizarla, cómo evaluar su idoneidad y darle el uso adecuado de acuerdo con el problema que se plantea» (CRUE-TIC \& REBIUN, 2009, p. 7)

Se pueden identificar, a partir de esta y de la mayor parte de las definiciones existentes en la literatura científica, cuatro sub-competencias, implicadas en el desarrollo de las competencias informacionales (CRUE-TIC \& REBIUN, 2009):

- Búsqueda de información: La persona es capaz de localizar y hacer una selección eficaz de la información necesaria dado el problema informacional concreto

- Evaluación de información: Capacidad para hacer una evaluación crítica de la calidad y pertinencia de las fuentes de información consultadas.

- Procesamiento de información: La persona está capacitada para extraer la información que realmente necesita a partir de las fuentes que ha seleccionado y evaluado y transformarla en conocimiento.

- Comunicación de información: Habilidades para comunicar y difundir la información procesada de manera eficaz a través de los canales más adecuados para la audiencia receptora.

Desde el nacimiento del concepto de competencias informacionales en los años 70 (Zurkowski, 1974), hasta la actualidad, ha habido una larga evolución del concepto y la materia propia de las competencias informacionales y de la forma en la que se evalúa su contenido. A partir de los años 90 las competencias informacionales entran en auge, y surgen numerosas instituciones e investigadores que aportan contenido y cuerpo propio a este concepto, generando estándares y normas que aportan indicadores sobre las competencias, sub-competencias y desempeños concretos incluidos dentro de las competencias informacionales.

La primera institución que lleva a cabo un esfuerzo en este sentido es la ALA (1989), que publica el informe "American Library Association Presidential Committee on Information Literacy: final report", en el que se comienza la senda de la estandarización y normativización de las competencias. En el informe, se definen cuatro componentes dentro de las competencias informacionales: habilidad para reconocer cuándo se necesita una información, y para localizar, 
evaluar y usar con eficacia la información requerida. Estos componentes, aunque con matices, han sido mantenidos como subcompetencias de la propia competencia general hasta la actualidad.

Después del informe de la ALA, a finales de la década de los años 90 y principios del siglo XXI, se comienza una carrera para crear un estándar válido de competencias informacionales. Así, varias asociaciones nacionales de bibliotecas lanzan sus normas que, como se indicó en el apartado teórico respectivo, tienen muchos puntos en común. En este ámbito, la Association of College \& Research Libraries (ACLR), parte integrante de la ALA, publica un informe sobre estándares y normas (2000). En el ámbito europeo, la Society of College, National and University Libraries (SCONUL) es la representante principal que publica un manual con los estándares y normas básicas (2001). También en el ámbito europeo se encuentra el Chartered Institute of Library and Information Proffesionals (CILIP), que desarrolla algunas normas agregadas a las anteriores (Abell et al., 2004). En el contexto del continente australiano, con la emergencia de investigadores destacados en este ámbito como Alan Bundy, Christine Bruce o Colin Lankshear, aparece el Australian and New Zealand Institute for Information Literacy (ANZIIL), que publica también sus propios estándares (2004).

De este modo, mientras que durante los años 90 se comienza a desarrollar el cuerpo teórico que compone este campo, es a finales de esta década y a principios de la nueva cuando se generaliza un corpus común aceptado globalmente.

En cuanto a la evaluación de las competencias informacionales, podríamos hablar de la evaluación de diferentes realidades. Por una parte, existen estudios que evalúan las competencias informacionales de los alumnos (Ozkul \& Kaya, 2009; Resnis, Gibson, \& Hartsell-Gundy, 2010; Rodríguez Conde, Olmos Migueláñez, Pinto Molina, Martínez Abad, \& García Riaza, 2011) y de los profesores (Bury, 2011; Probert, 2009; Williams \& Coles, 2007; Williams \& Wavell, 2007). En estos casos el instrumento de evaluación empleado suele ser de elaboración propia, o adaptado de otros estudios. La falta de instrumentos de evaluación estandarizados dificulta mucho la comparabilidad entre estudios y regiones geográficas (Hernández Ramos, Martínez Abad, García Peñalvo, Herrera García, \& Rodríguez Conde, 2013; Schmidt, 2008). Por otra parte, también se encuentran varios trabajos que se proponen el análisis de los programas formativos existentes de integración de las competencias informacionales (Emmett \& Emde,
2007; Gratch Lindauer, 2006; Marzal GarcíaQuismondo, 2010), aunque estos últimos no se corresponden con el tópico aquí estudiado.

Volviendo al tema de la evaluación de las competencias informacionales en el profesorado y alumnado, surge el problema de su medida. Mientras que en la mayor parte de los casos se emplean herramientas de elaboración propia o adaptadas de otras no estandarizadas (Ferguson, Neely, \& Sullivan, 2006; Probert, 2009; Resnis et al., 2010). No se tiene clara la preferencia entre escalas de autopercepción de la competencia (Kurbanoglu, Akkoyunlu, \& Umay, 2006; Ozkul \& Kaya, 2009; Pinto Molina, 2009; Williams \& Wavell, 2007) o de actitudes hacia la misma (Bury, 2011; Richter, Naumann, \& Groeben, 2000). Lo que se pone en evidencia es que no se localizan escalas con un estudio psicométrico sistemático y completo, y las que se encuentran no obtienen los ajustes esperados (Rodríguez Conde, Olmos Migueláñez, \& Martínez Abad, 2012). Por otro lado, no se localizan en la literatura escalas de evaluación de la competencia real, siendo lo más extendido y aceptado las escalas de autoeficacia que, aunque poseen una base que les aporta validez, deben ser interpretadas con las pertinentes reticencias.

Por otro lado, dentro de esta línea de investigación, se localizan estudios que aplican programas formativos a una muestra de alumnos concreta (Freeman \& Lynd-Balta, 2010; Hernández Hernández, 2010; Mackey \& Ho, 2005; Warschauer, 2007). Suelen tratarse de estudios de caso en los que se aportan evidencias sobre la eficacia del programa en concreto, que suele ser diseñado por el propio equipo de investigación a partir de los estándares aportados por alguna de las instituciones señaladas más arriba. La evaluación de los programas se realiza con instrumentos no estandarizados de manera generalizada. Así, a pesar de que se adivina una sistematización de los contenidos que se incluyen dentro de las competencias informacionales, la investigación sigue adoleciendo de instrumentos de evaluación de las mismas que resulten comparables y con unas cualidades psicométricas aceptables.

Otro aspecto importante a tener en cuenta es el nivel educativo en el que se desarrollan las propias competencias informacionales. En este ámbito se localizan principalmente estudios enfocados en la etapa universitaria de los estudiantes (Hernández Serrano \& Fuentes Agustí, 2011; Hernández Serrano, 2009; Ozkul \& Kaya, 2009; Pinto Molina, 2009; Resnis et al., 2010), mientras que los estudios relacionados con las etapas de educación básica u obligatoria, son 
menos numerosas y más centradas en desempeños concretos de las competencias informacionales, principalmente la búsqueda de información (Fuentes Agusti \& Monereo, 2008; Head \& Eisenberg, 2009; Pifarré, Sanuy, Vendrell, \& Gòdia, 2009).

Dada esta situación de partida, y teniendo en cuenta los estudios previos llevados a cabo por el grupo (Rodríguez Conde et al., 2012; Rodríguez Conde et al., 2011) en torno a la escala de evaluación de competencias informacionales autopercibidas IL-HUMASS (Information Literacy Humanities Social Sciences survey) (Pinto Molina, 2009), el presente estudio tiene como objetivo profundizar la indagación de las propiedades psicométricas de dicha escala para comprender mejor las dimensiones percibidas por los sujetos en cuanto a los desempeños propios de las competencias informacionales.

Dado que los resultados empíricos sobre la naturaleza y dimensiones del constructo competencias informacionales obtenidos en los estudios señalados, que aplican principalmente técnicas factoriales exploratorias y confirmatorias, no aportan evidencias consistentes, se cree conveniente realizar un estudio multidimensional previo como el aquí presentado. El escalamiento multidimensional se toma en esta investigación como una técnica que aseguran la validez de contenido de escalas que miden constructos poco definidos. Así, se tratarán de aportar evidencias claras acerca de la existencia de factores latentes alternativos a las cuatro dimensiones teóricas de las competencias informacionales comúnmente aceptadas, que estén interactuando con éstas y que afecten su comportamiento multidimensional.

Para tratar de dar respuesta a dicho objetivo, se sistematizará la observación de las diferencias y similitudes más importantes entre las variables de la escala IL-HUMASS a través de un escalamiento multidimensional.

\section{Metodología}

\section{Diseño de la investigación}

Se plantea un diseño no experimental, de tipo ex post facto, a través de estudios de encuesta (Morales Vallejo, 2000). No se pretende manipular algunas variables para observar el comportamiento individual y conjunto, sino medir las variables en su contexto natural y estudiar su distribución para explicar una realidad que se desconoce de partida.

\section{Población y muestra}

La población de partida es el futuro profesorado de educación secundaria, estudiante del Máster profesionalizante de profesor en Educación Secundaria en España. Se obtiene una muestra no probabilística de manera incidental a partir de los sujetos que están estudiando el citado Máster durante los cursos 2009/2010 y 2010/2011 en la Comunidad Autónoma de Castilla y León. La muestra final obtenida es de 402 sujetos a partir del conglomerado formado por los estudiantes de la Universidad de Salamanca, de entre las 4 universidades públicas que imparten el Máster de profesorado. En cuanto a la descripción de la muestra, se obtiene para la variable sexo un $62 \%$ de hombres y un $38 \%$ de mujeres, y una edad media de 26,16 años $\left(S_{x}=5,54\right)$.

\section{Variables y escala de medida}

Las variables que intervienen en el estudio son los 26 ítems que conforman el instrumento ILHUMASS, todos ellos tipo Likert, con opciones de respuesta de 1 a 9, correspondiendo 1 con un nivel autopercibido muy bajo o nulo y 9 con un nivel autopercibido muy alto o máximo. Cabe recordar, que el instrumento posee una estructura factorial conocida (Rodríguez Conde et al., 2012), compuesta por cuatro dimensiones: búsqueda de información, evaluación de información, procesamiento de información y comunicación de información.

Se evidencia que en la medida de actitudes, los cuestionarios basados en escalas de respuesta son la técnica de recogida de datos más habitual (Hernández Pina, 2009; Hernández Ramos, Martínez Abad, García Peñalvo, Herrera García, \& Rodríguez Conde, 2014), dado que es el método mejor fundamentado, más estudiado y contrastado (Morales Vallejo, 2000; Tejedor Tejedor, García-Valcarcel, \& Prada, 2009).

Además, no se debe olvidar que se trabaja con una escala de evaluación en la competencia informacional de autoeficacia, haciendo la consideración de autoeficacia como "el conjunto de juicios de cada individuo sobre las capacidades propias para organizar y ejecutar las acciones requeridas en el manejo de posibles situaciones específicas" (Blanco Blanco, 2010, pp. 1-2). Cuando se valora una competencia compleja mediante técnicas expost-facto, en una muestra no probabilística e intencional de futuros profesores de educación secundaria, como en este caso, de manera transversal, el valor empírico de la medida de autopercepción, a través de un cuestionario de autoinforme, se avala en gran cantidad de investigaciones (Bandura \& Locke, 2003; Eastman \& Marzillier, 1984; Vancouver, 2005). Así, las evidencias obtenidas en la literatura científica apoyan el valor predictivo de la autopercepción en el ámbito concreto de las 
Ciencias de la Educación (Lent, 1994; Robbins et al., 2004; Rottinghaus, Larson, \& Borgen, 2003).

\section{Análisis de datos}

De cara a realizar una exploración previa de las tendencias de respuesta de los sujetos a las dimensiones de la escala, se realiza un escalamiento multidimensional mediante el método ALSCAL con los 26 ítems del cuestionario. El escalamiento multidimensional se implementa forzando el análisis a dos dimensiones. Posteriormente se lleva a cabo un análisis gráfico de los resultados obtenidos, tanto por ítems individualmente, como por cada una de las 4 dimensiones teóricas.

\section{Resultados}

De acuerdo a lo expuesto precedentemente, se decide aplicar un escalamiento multidimensional para comprobar las diferencias y similitudes entre las variables en dos dimensiones. Se forzará el modelo a dos dimensiones para poder representar en un plano la matriz de disimilitudes obtenida y facilitar así su interpretación.

Se seleccionan, por tanto, como variables del escalamiento los 26 ítems de la escala inicial. Se calcula la matriz de distancias entre las variables a partir de la distancia euclídea, y en el modelo se selecciona una escala de medida ordinal.

Aplicado el modelo, se obtiene un stress para la matriz calculada de 0,11666 , y una correlación al cuadrado entre distancias y disparidades de 0,95896. Así, podemos pensar que el modelo tiene un buen ajuste, dado el alto índice de correlación al cuadrado y el bajo valor para el stress. En cuanto a la matriz obtenida para las dos dimensiones calculadas, se puede observar en la Tabla 1.

El diagrama de dispersión, que se puede observar en el Gráfico 1, muestra cómo se distribuyen las variables en estas dos dimensiones.

La primera dimensión, hace referencia al nivel de familiaridad con el desempeño de la actividad que propone el ítem, teniendo unos valores más bajos aquellos ítems con los que el sujeto tiene una familiaridad menor. Así, el ítem 17 se relaciona con una actividad poco habitual, como emplear gestores de referencias bibliográficas, que es un campo prácticamente desconocido para el profesorado universitario. También tienen valores bajos en esta primera dimensión el ítem 16,
Tabla 1. Coordenadas de la matriz de dimensiones

\begin{tabular}{ccc}
\hline \multirow{2}{*}{ ÍTEMS } & \multicolumn{2}{c}{ Dimensión } \\
\cline { 2 - 3 } & 1 & 2 \\
\hline ÍTEM_01 & 1,089 & 0,0482 \\
ÍTEM_02 & $-0,1295$ & 0,2848 \\
ÍTEM_03 & 0,8298 & 0,1715 \\
ÍTEM_04 & $-0,2862$ & 0,3795 \\
ÍTEM_05 & 1,0759 & 0,2005 \\
ÍTEM_06 & 1,0087 & 0,3338 \\
ÍTEM_07 & 0,0352 & 0,1308 \\
ÍTEM_08 & $-2,3612$ & 0,4992 \\
ÍTEM_09 & 0,5382 & 0,1146 \\
ÍTEM_10 & 1,4116 & $-0,2112$ \\
ÍTEM_11 & $-0,301$ & $-0,3232$ \\
ÍTEM_12 & 0,4014 & $-0,0416$ \\
ÍTEM_13 & 0,4947 & $-0,3984$ \\
ÍTEM_14 & 1,442 & 0,0603 \\
ÍTEM_15 & 1,3066 & 0,0374 \\
ÍTEM_16 & $-2,1034$ & 0,7649 \\
ÍTEM_17 & $-3,5417$ & $-0,2442$ \\
ÍTEM_18 & $-1,5653$ & 0,8377 \\
ÍTEM_19 & $-0,0459$ & 1,3398 \\
ÍTEM_20 & 0,2562 & $-0,4093$ \\
ÍTEM_21 & $-0,8798$ & $-2,4867$ \\
ÍTEM_22 & 0,979 & $-0,3344$ \\
ÍTEM_23 & 0,3758 & $-0,5415$ \\
ÍTEM_24 & $-1,2448$ & $-0,7258$ \\
ÍTEM_25 & 1,1052 & 0,3241 \\
ÍTEM_26 & 0,1097 & 0,189 \\
\hline & & \\
& &
\end{tabular}

referido al empleo de bases de datos, o el ítem 8 , referido al empleo de estrategias de búsqueda de información. Cabe destacar, por tanto, que cuanta más baja es la puntuación asignada a los ítems en esta primera dimensión menor familiaridad tienen las personas con el propio ítem. En este sentido, los ítems 8 y 17 obtienen puntuaciones z extremas $(\mathrm{z}<-1.96)$ por la cola inferior de la distibución.

En cuanto a la segunda dimensión, hace referencia al componente de la actividad en un continuo que va de lo meramente instrumental (el ítem 19 se refiere a instalar programas informáticos) a lo meramente cognitivo (el ítem 21 se refiere a saber comunicar en otros idiomas). El gráfico 2 indica que el ajuste del modelo es adecuado. Las puntuaciones altas en esta dimensión se refieren a un componente más instrumental y las puntuaciones bajas a un componente más cognitivo. En esta segunda 
Figura 1. Representación del modelo de distancias euclídeas

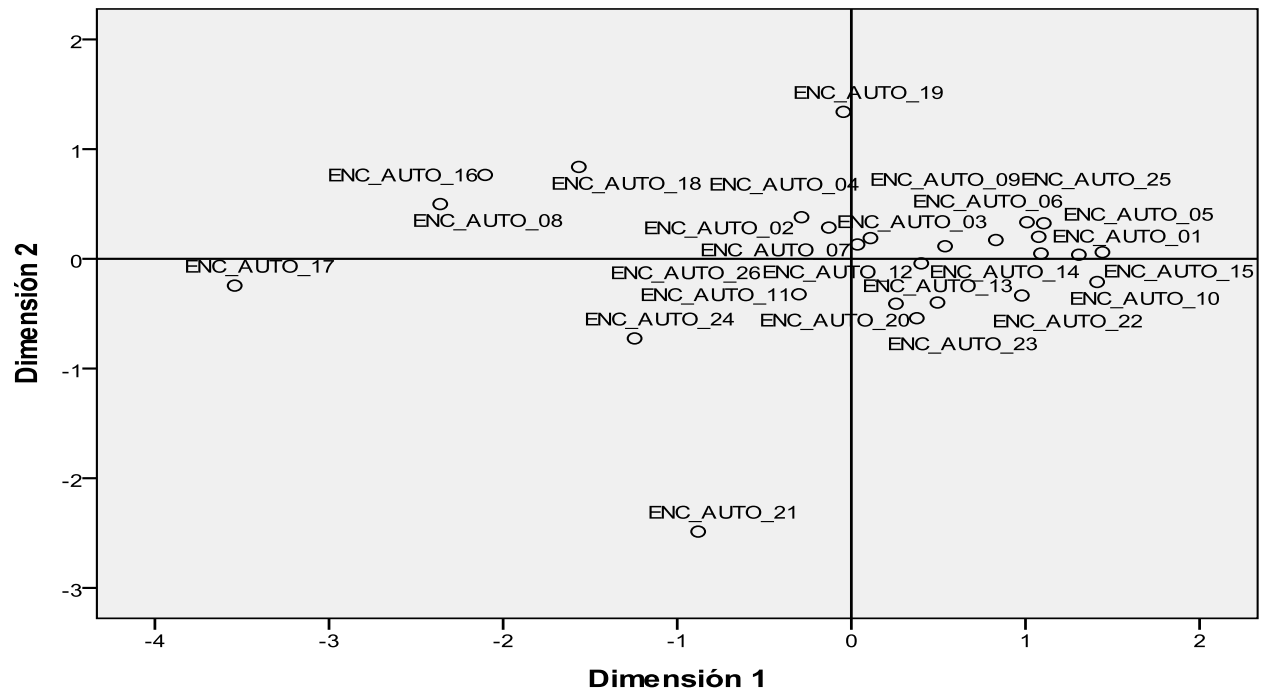

dimensión no existe ningún ítem con puntuaciones tan extremas como anteirormente debido, en este caso, a que los ítems contienen dentro de su contenido un componente ecléctico, esto es, ni completamente cognitivo no completamente instrumental.

Figura 2. Ajuste lineal del modelo de escalamiento multidimensional

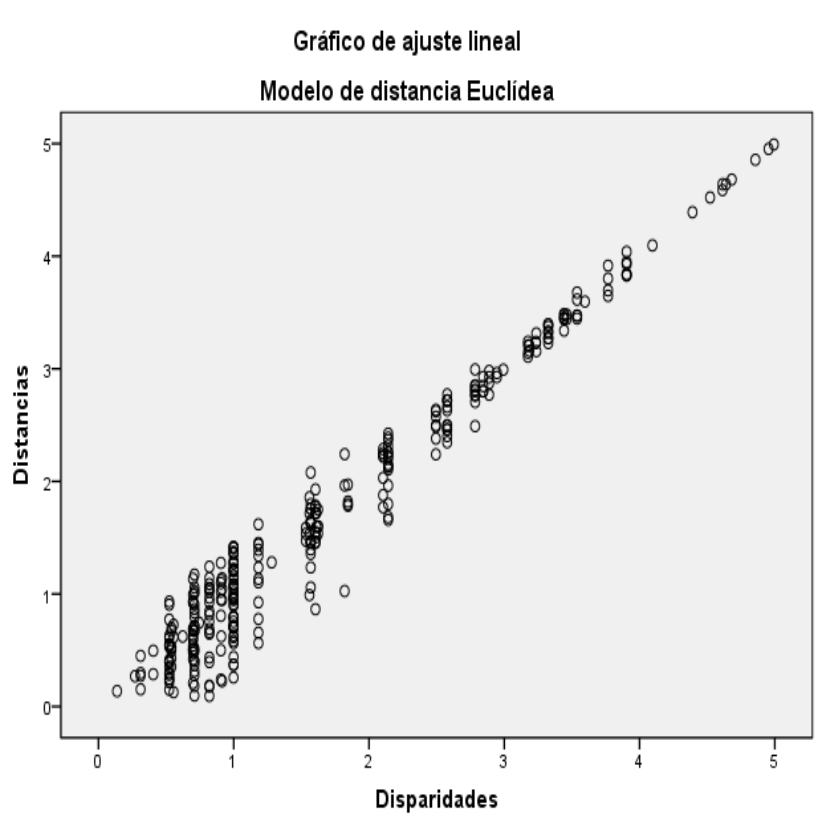

Si llevamos a cabo la representación del modelo separando las variables por sus dimensiones teóricas (Gráfico 3) podemos hacer un análisis más detallado.

Se observa cómo las variables de búsqueda de información son de un ámbito más instrumental y la familiaridad del futuro profesorado con las mismas es alta. Por otro lado, las variables de evaluación también son familiares, pero son más de tipo cognitivo.

En lo que respecta a las variables de procesamiento, se observan las diferencias entre las dos variables de tipo cognitivo identificadas en el primer estudio, que son muy familiares, con las de tipo instrumental, que son menos familiares. Por último, las variables relacionadas con la comunicación se distribuyen de manera más dispersa.

Figura 3. Representación del modelo de distancias euclídeas en función de la dimensión teórica

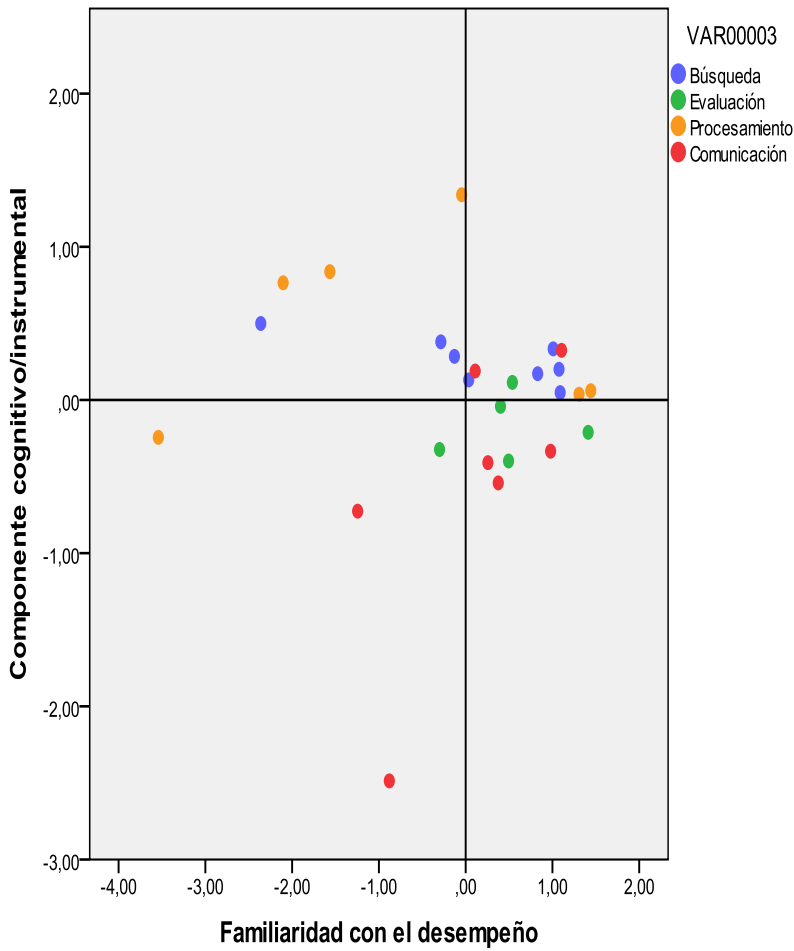




\section{Discusión y conclusiones}

Como se ha demostrado en la literatura científica analizada, la evaluación de competencias en general, y de competencias informacionales en particular, se lleva a cabo mediante procedimientos parciales y no estandarizados (Ferguson et al., 2006; Probert, 2009; Resnis et al., 2010). Por otro lado, la elección de los investigadores suele ser la de elaborar ad-hoc escalas para realizar este tipo de evaluaciones de la competencia autopercibida (Kurbanoglu et al., 2006; Ozkul \& Kaya, 2009; Pinto Molina, 2009; Williams \& Wavell, 2007), con lo que se dificulta la comparación entre los efectos de unos programas y otros y, con ello, el desarrollo científico de la disciplina.

Esto se debe, a nuestro juicio, a la falta de escalas con unas propiedades psicométricas adecuadas, inclusivas y de calidad. Las evidencias obtenidas en esta investigación muestran cómo es necesario el desarrollo y mejora de escalas como la IL-HUMASS (Pinto Molina, 2009), aquí estudiada.

Se pone en evidencia la posibilidad de mejorar esta escala atendiendo, no sólo al eje de las dimensiones teóricas relativas a la búsqueda de información, evaluación de información, procesamiento de información y comunicación de información, sino, además, a un segundo eje que contemple distintos niveles de dificultad de cada desempeño incluido en las dimensiones teóricas y distintos desempeños relacionados con aspectos cognitivos e instrumentales de la dimensión. Teniendo en cuenta estos dos aspectos dentro de cada dimensión se podrán construir escalas para la evaluación de competencias informacionales autopercibidas más completas y adecuadas.

Para apoyar la importancia y credibilidad de estas afirmaciones, contamos como puntos fuertes tanto la consistencia y nivel de sistematicidad, que aportan a la escala IL-HUMASS los estudios realizados alrededor de la misma (Pinto Molina \& Puertas Valdeiglesias, 2012; Rodríguez Conde et al., 2012; Rodríguez Conde et al., 2011), como los buenos índices de varianza explicada como de stress obtenidos en el estudio. También se pueden señalar algunos puntos débiles, sobre todo los que tienen que ver con la naturaleza del instrumento aplicado para la recogida de información. A pesar de que la evidencia empírica da soporte a la relación entre las medidas de autopercepción y el nivel real (Lent, 1994; Robbins et al., 2004; Rottinghaus et al., 2003), no debemos olvidar este hecho, y debemos ser cautelosos con el empleo de escala de autopercepción para la evaluación de una competencia básica.

Revista Iberoamericana de Diagnóstico y Evaluación - e Avaliação Psicológica. RIDEP · Nº42 · Vol.2 · 39-48 2016
Las líneas de investigación que emanan del estudio tienen que ver, principalmente, con el apoyo que la presente investigación presta a futuros intentos de diseño y adaptación de escalas de evaluación de competencias básicas autopercibidas, especialmente en el campo de las competencias informacionales, ya que aporta un marco teórico sobre el que sustentar la elaboración de los ítems dentro de cada dimensión o sub-competencia.

\section{Agradecimientos}

Spanish National R+D+i Project, 2012: Evaluación, Formación e Innovación sobre Competencias clave en Educación Secundaria: TIC, Competencia Informacional y Resolución de Conflictos (EFI-CINCO). Ref: EDU2012-34000.

Spanish National R+D+i Project, 2009: Evaluación de competencias clave y Formación de Profesorado de Educación Secundaria: TIC, ALFIN y Convivencia Escolar (EF-TALCO). Ref: EDU2009-08753

\section{Referencias}

Abell, A., Armstrong, C., Boden, D., Town, J. S., Webber, S., \& Woolley, M. (2004). Alfabetización en información: La definición de CILIP (UK). Boletín de la Asociación Andaluza de Bibliotecarios, (77), 79-84.

ALA/ACRL (2000). Information literacy competency standards for higher education. Chicago IL: ACRL.

American Library Association. (1989). American Library Association Presidential Committee on Information Literacy: Final report. Chicago: American Library Association.

Area Moreira, M. (2001). Educar en la sociedad de la información. Bilbao: Desclée De Brouwer.

Bandura, A., \& Locke, E. A. (2003). Negative self-efficacy and goal effects revisited. The Journal of Applied Psychology, 88(1), 87-99.

Blanco Blanco, Á. (2010). Creencias de autoeficacia de estudiantes universitarios: Un estudio empírico sobre la especificidad del constructo. Revista Electrónica de Investigación y Evaluación Educativa, 16(1), $1-28$.

Bundy, A., \& ANZIIL. (2004). Australian and New Zealand information literacy framework principles, standards and practice. Adelaide 
(Australia): Australian and New Zealand Institute for Information Literacy. Recuperado a partir de http://www.anziil.org/resources/Info $\% 201$ lit $\% 2$ 02nd\%20edition.pdf

Bury, S. (2011). Faculty attitudes, perceptions and experiences of information literacy: A study across multiple disciplines at York University, Canada. Journal of Information Literacy, 5(1), 44-64.

Cassity, E., \& Ang, I. (2006). Industry partnerships and the «Knowledge Society»: the australian experience. Minerva: A Review of Science, Learning and Policy, 44(1), 47-63.

Castells, M. (1999). La Era de la información: economía, sociedad y cultura. La sociedad Red (Vol. 1). Madrid: Alianza Editorial.

Cebrián Herreros, M. (2009). La sociedad de la información y del conocimiento en los países nórdicos. Barcelona: Gedisa.

CRUE-TIC, \& REBIUN. (2009). Competencias informáticas e informacionales en los estudios de grado. Conferencia de Rectores de Universidades Españolas

Delors, J. (1997). La educación encierra un tesoro: Informe a la UNESCO de la Comisión Internacional de la Educación para el Siglo $X X I$. Madrid: UNESCO.

Diario oficial de la Unión Europea. (2006). Recomendación del Parlamento Europeo y del Consejo de 18 de diciembre de 2006 sobre las competencias clave para el aprendizaje permanente. Bruselas: Parlamento Europeo y Consejo de la Unión europea.

Eastman, C., \& Marzillier, J. S. (1984). Theoretical and methodological difficulties in Bandura's self-efficacy theory. Cognitive Therapy and Research, 8(3), 213-229.

Emmett, A., \& Emde, J. (2007). Assessing information literacy skills using the ACRL standards as a guide. Reference Services Review, 35(2), 210-229.

Ferguson, J., Neely, T., \& Sullivan, K. (2006). A baseline information literacy assessment of biology students. Reference \& user services quarterly, 46(2), 61-71.

Freeman, E., \& Lynd-Balta, E. (2010). Developing information literacy skills early in an undergraduate curriculum. College Teaching, 58(3), 109-115.
Fuentes Agusti, M., \& Monereo, C. (2008). Como buscan información en Internet los adolescentes. Investigación en la escuela, (64), 45-58.

García Carrasco, J. (2009). Las formas de alfabetización cultural en la sociedad de la información. Teoría de la Educación: Educación y Cultura en la Sociedad de la Información, 10(1), 49-75.

Gratch Lindauer, B. (2006). Los tres ámbitos de evaluación de la alfabetización informacional. Anales de Documentación, 9, 69-81.

Head, A. J., \& Eisenberg, M. (2009). How college students seek information in the digital age. Washington D.C.: University of Washington.

Hernández Hernández, C. J. (2010). Un plan de formación en competencias de información a través de aulas virtuales: Análisis de una experiencia con alumnado universitario. Revista de Universidad y Sociedad del Conocimiento, 7(2), 50-62.

Hernández Pina, F. (2009). Aprendizaje y competencia. Una nueva mirada. Revista Española de Orientación y Psicopedagogía, 20(3), 312-319.

Hernández Ramos, J. P., Martínez Abad, F., García Peñalvo, F. J., Herrera García, M. E., \& Rodríguez Conde, M. J. (2014). Teachers' attitude regarding the use of ICT. A factor reliability and validity study. Computers in Human Behavior, 31, 509-516. doi:10.1016/j.chb.2013.04.039

Hernández Serrano, M. J. (2009). Estrategias de búsqueda de información para la generación de conocimiento en red. Salamanca: Ediciones Universidad de Salamanca.

Hernández Serrano, M. J., \& Fuentes Agustí, M. (2011). Aprender a informarse en la red: Son los estudiantes eficientes buscando y seleccionando la información? Teoría de la Educación: Educación y Cultura en la Sociedad de la Información, 12(1), 47-78.

Kurbanoglu, S. S., Akkoyunlu, B., \& Umay, A. (2006). Developing the information literacy self-efficacy scale. Journal of Documentation, 62(6), 730-743.

Lent, R. W. (1994). Toward a unifying social cognitive theory of career and academic interest, choice, and performance. Journal of Vocational Behavior, 45(1), 79-122. 
Mackey, T., \& Ho, J. (2005). Implementing a convergent model for information literacy: Combining research and web literacy. Journal of Information Science, 31(6), 541-555.

Martorell, M. C., Gónzalez, R., Aloy, M., \& Ferris, M. C. (1995). Socialización y conducta prosocial. Revista Iberoamericana de Diagnóstico y Evaluación - e Avaliação Psicológica, 1(1), 73-102.

Marzal García-Quismondo, M. Á. (2010). La evaluación de los programas de alfabetización en información en la educación superior: Estrategias e instrumentos. Revista de Universidad y Sociedad del Conocimiento, 7(2), 28-38.

McLuhan, M. (1987). Understanding media: The extensions of man. London: Routledge.

Morales Vallejo, P. (2000). Medición de actitudes en psicología y educación: Construcción de escalas y problemas metodológicos. Madrid: Universidad Pontificia Comillas.

OCDE. (2004). Completing the foundation for lifelong learning. An OECD survey of upper secondary schools. Centre For Educational Research And Innovation.

Ozkul, H., \& Kaya, H. (2009). The views of nursing students about their own information literacy. New Educational Review, 19(3-4), 45-57.

Pifarré, M., Sanuy, J., Vendrell, C., \& Gòdia, S. (2009). Internet en la educación secundaria: Pensar, buscar y construir conocimiento en la red. Lleida: Milenio.

Pinto Molina, M. (2009). Design of the ILHUMASS survey on information literacy in higher education: A self-assessment approach. Journal of Information Science, 36(1), 86103.

Pinto Molina, M., \& Puertas Valdeiglesias, S. (2012). Autoevaluación de la competencia informacional en los estudios de psicología desde la percepción del estudiante. Anales de Documentación, 15(2), 1-15.

Prensky, M. (2009). Homo sapiens digital: From digital immigrants and digital natives to digital wisdom. Innovate: Journal of Online Education, 5(3).

Probert, E. (2009). Information literacy skills: Teacher understandings and practice.
Computers \& Education, 53(1), 24-33. doi:10.1016/j.compedu.2008.12.018

Resnis, E., Gibson, K., \& Hartsell-Gundy, A. (2010). Information literacy assessment: A case study at Miami University. New Library World, 111(7/8), 287-301.

Richter, T., Naumann, J., \& Groeben, N. (2000). Attitudes toward the computer: Construct validation of an instrument with scales differentiated by content. Computers in Human Behavior, 16(5), 473-491. doi:10.1016/S0747-5632(00)00025-X

Robbins, S. B., Lauver, K., Le, H., Davis, D., Langley, R., \& Carlstrom, A. (2004). Do psychosocial and study skill factors predict college outcomes? A metaanalysis. Psychological Bulletin, 130(2), 261-288.

Rodríguez Conde, M. J., Olmos Migueláñez, S., \& Martínez Abad, F. (2012). Propiedades métricas y estructura dimensional de la adaptación española de una escala de evaluación de competencia informacional autopercibida (IL-HUMASS). Revista de Investigación Educativa, 30(2), 347-365.

Rodríguez Conde, M. J., Olmos Migueláñez, S., Pinto Molina, M., Martínez Abad, F., \& García Riaza, B. (2011). Informational literacy and information and communication technologies use by secondary education students in spain: A descriptive study. Contemporary Issues in Education Research (CIER), 4(4), 1-12.

Rottinghaus, P. J., Larson, L. M., \& Borgen, F. H. (2003). The relation of self-efficacy and interests: A meta-analysis of 60 samples. Journal of Vocational Behavior, 62(2), 221236.

Schmidt, C. M. (2008). Construcción de un cuestionario de emociones positivas en población entrerriana. Revista Iberoamericana de Diagnóstico y Evaluación - e Avaliação Psicológica, 2(26), 117-139.

SCONUL. (2001). Information skills in higher education: A SCONUL position paper. London: SCONUL.

Tejedor Tejedor, F. J., García-Valcarcel, A., \& Prada, S. (2009). Medida de actitudes del profesorado universitario hacia la integración de las TIC. Comunicar, (33), 115-124. 
UNESCO. (2005). Towards knowledge societies. Paris: UNESCO.

Valimaa, J., \& Hoffman, D. (2008). Knowledge society discourse and higher education. Higher Education: The International Journal of Higher Education and Educational Planning, 56(3), 265-285.

Vancouver, J. B. (2005). The depth of history and explanation as benefit and bane for psychological control theories. The Journal of Applied Psychology, 90(1), 38-52.

Warschauer, M. (2007). Information literacy in the laptop classroom. Teachers College Record, 109(11), 2511-2540.

Williams, D., \& Coles, L. (2007). Teachers' approaches to finding and using research evidence: An information literacy perspective. Educational Research, 49(2), 185-206.

Williams, D., \& Wavell, C. (2007). Secondary school teachers' conceptions of student information literacy. Journal of Librarianship and Information Science, 39(4), 199-212.

Zurkowski, P. (1974). The information service environment: Relationships and priorities. Washington D.C.: National Commission on Libraries and Information Science. 